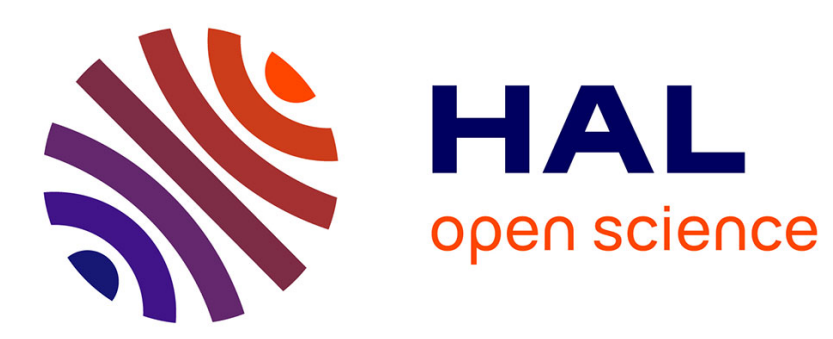

\title{
DC SQUID Amplifier for NMR Experiments on Small Samples
}

O. Hubert, Y. Monfort, J. Lepaisant, C. Gunther, Daniel Bloyet

\section{To cite this version:}

O. Hubert, Y. Monfort, J. Lepaisant, C. Gunther, Daniel Bloyet. DC SQUID Amplifier for NMR Experiments on Small Samples. Journal de Physique IV Proceedings, 1996, 06 (C3), pp.C3-411-C3416. 10.1051/jp4:1996362 . jpa-00254280

\section{HAL Id: jpa-00254280 https://hal.science/jpa-00254280}

Submitted on 1 Jan 1996

HAL is a multi-disciplinary open access archive for the deposit and dissemination of scientific research documents, whether they are published or not. The documents may come from teaching and research institutions in France or abroad, or from public or private research centers.
L'archive ouverte pluridisciplinaire HAL, est destinée au dépôt et à la diffusion de documents scientifiques de niveau recherche, publiés ou non, émanant des établissements d'enseignement et de recherche français ou étrangers, des laboratoires publics ou privés. 


\title{
DC SQUID Amplifier for NMR Experiments on Small Samples
}

\author{
O. Hubert, Y. Monfort, J. Lepaisant, C. Gunther and D. Bloyet \\ Groupe de Recherches en Informatique, Image et Instrumentation de Caen (G.R.E.Y.C.), URA 1526 \\ CNRS, ISMRA, 6 Boulevard du Maréchal Juin, 14050 Caen Cedex, France
}

\begin{abstract}
Low noise preamplifiers are required for the detcction of the magnctic resonance of small size samples or at low magnetic ficlds. According to calculations based on the " $e_{11}, i_{n}$ " model. SQULD based amplifiers arc more suitable to NMR experiments on small samples than semiconductor amplifiers. We propose an amplification method by frequency transposition using a DC SQUID. Such a system can be run orer a broad range of frequency as the noise impedance of the stages subsequent to the SQUID is continually optimised. The measurements are in good agreement with our theoretical model, based on a simplified voltage to flux SQUID characteristic.
\end{abstract}

Due to their high sensitivity, DC SQUIDs have been involved for ten years in magnetic resonance experiments where the magnetisation level of the samples is low, in particular in the case of low magnetic field experiments or NMR detection from small samples (or thin films) [1] [2] [3]. In section 1, the performances of DC SQUID and semiconductor amplifiers are compared when they are involved in NMR measurements on various sizes cooled samples. Our calculations, based on the " $e_{n}, i_{n}$ " noise model in the low frequency range, take different configurations of the input stage into account. In section 2 , a new method of amplification derived from the flux modulation techniques is presented [4] and a simplified model explaining qualitatively our experimental results is proposed.

\section{CONFIGURATIONS USED TO DETECT NMR SIGNALS}

\subsection{Noise characteristics of semiconductor and DC SQUID amplifier}

Two classes of low noise amplifiers have been developed to sense weak signals : the semiconductor and the SQUID amplifiers. Their main figures of merit are the $e_{n} i_{n}$ product and the $e_{n} / i_{n}$ optimal input impedance. So we are going to compare their respective performances before studying the configurations adapted to detect NMR signals. Our study is restricted to frequencies ranging from $50 \mathrm{kHz}$ to $1 \mathrm{MHz}$

The widely used semiconductor amplifiers (FET) have a high optimal input impedance (few $10^{4} \Omega$ ), their best noise energy is about $10^{-24} \mathrm{~V} / \mathrm{A} / \mathrm{Hz}$. Their resolution is optimal in the narrow frequency band $100 \mathrm{z}-$ $500 \mathrm{kHz}$ and for operating temperatures close to the room temperature (Si JFET). Lower temperatures can be reached by GaAs MESFETs but these devices show flicker noise under $1 \mathrm{MHz}$.

It is more complex to make use of a DC SQUID amplifier than a semiconductor one as the SQUID operates in a cryogenic environment and is drastically influenced by electromagnetic disturbances. For a coupled DC SQUID (Fig. 1) having an inductance $L_{s}$, a critical current $2 I_{c}$, a junction resistance $R$, a dynamic resistance $R_{d}$, a coupling inductance $L_{i}$, a coupling factor $\alpha_{c}=M / \sqrt{L_{s} L_{i}}$ and $2 L_{s} I_{c} / \Phi_{0} \approx 1$, the noise characteristics are given by [5]:

$$
\begin{aligned}
& \overline{\mathrm{e}_{\mathrm{n}}{ }^{2}}=\omega^{2} \mathrm{~L}_{\mathrm{i}} \mathrm{L}_{\mathrm{s}} \mathrm{k}_{\mathrm{B}} \mathrm{T}\left(16 / \alpha_{\mathrm{c}}^{2}+11 \alpha_{\mathrm{c}}^{2}-24\right) / \mathrm{R}=\left(\mathrm{K}_{3} \omega\right)^{2} \\
& \overline{\mathrm{i}_{\mathrm{n}}{ }^{2}}=16 \mathrm{k}_{\mathrm{B}} \mathrm{TL} \mathrm{L}_{\mathrm{s}} / \alpha_{\mathrm{c}}^{2} \mathrm{RL} \mathrm{L}_{\mathrm{i}} \text { and } \mathrm{C}=\mathrm{j} \mathrm{C}_{\mathrm{I}}=\overline{\mathrm{e}_{\mathrm{n}} \mathrm{i}_{\mathrm{n}}^{*}} / \sqrt{\overline{\mathrm{e}_{\mathrm{n}}{ }^{2} \mathrm{i}_{\mathrm{n}}{ }^{2}}}=-\mathrm{j}\left(1+2 \alpha_{\mathrm{c}}^{2} /\left(3 \alpha_{\mathrm{c}}^{2}-4\right)^{2}\right)^{-1 / 2}
\end{aligned}
$$


where $K_{3}, i_{n}$ and $C$ are independent of $\omega$ and $C$ is the correlation term between $e_{n}$ and $i_{n}$. The optimal noise impedance of the DC SQUID is deduced from the expressions of $e_{n}$ and $i_{n}$ : $e_{n} / i_{n}=\omega L_{i}\left(11 \alpha_{c}^{4} / 16-3 \alpha_{c}^{2} / 2+1\right)^{1 / 2}$. Numerical evaluations give for a commercial dc SQUID (Conductus SQD1002) $: \mathrm{K}_{3}=5.3810^{-20} \mathrm{SI}, \mathrm{i}_{\mathrm{n}}=0.594 \mathrm{pA} / \sqrt{\mathrm{Hz}}$ and $\mathrm{C}_{\mathrm{I}}=-0.880$ at $\mathrm{T}=4.2 \mathrm{~K}$. The product $\mathrm{e}_{\mathrm{n}} \mathrm{i}_{\mathrm{n}}$ and the optimal input impedance are respectively below $10^{-25} \mathrm{VA} / \mathrm{Hz}$ and $1 \Omega$ in the range $50 \mathrm{kHz}-1 \mathrm{MHz}$. So, DC SQUID amplifiers are more sensitive than semiconductor amplifiers but they have to be coupled to a low impedance generator.

\subsection{Coupling of a preamplifier to a NMR coil}

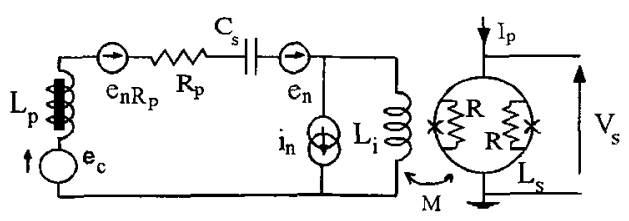

Figure 1 : Coupling of a NMR coil to a DC SQUID amplifier.

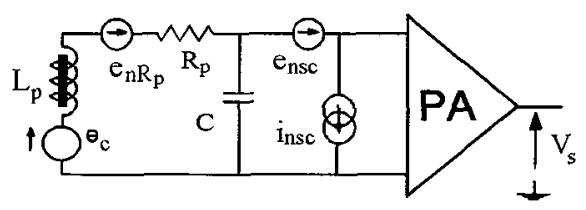

Figure 2 : Coupling of a NMR coil to a semiconductor amplifier.

\subsubsection{Semiconductor amplifier : selective configuration}

As the optimal input impedance of the semiconductor amplifiers is high, an efficient way to obtain the best signal to noise ratio is to use a tank circuit. A classical coupling of the receiver to the NMR coupling coil is shown in figure 2. The NMR signal induced in the pick-up coil by the nuclear spins is represented by a voltage generator $e_{c}$ and the circuit losses by a resistance $R_{p}$ which generates a thermal noise density $\overline{\mathrm{e}_{\mathrm{nRp}}^{2}}=4 \mathrm{k}_{\mathrm{B}} \mathrm{TR}_{\mathrm{p}} . \mathrm{e}_{\mathrm{nsc}}$ and $\mathrm{i}_{\mathrm{nsc}}$ are two equivalent noise generators resulting from the preamplifier (PA) noise. The best configuration for NMR experiment depends obviously on the sample volume. The sample geometry is a cylinder with a fixed cross section and a variable height. The sample contains $n_{\text {sple }}$ copper cylindrical wires of length $h_{\text {sple }}$ and radius $b$ and is cooled at $4.2 \mathrm{~K}$. So the pick-up coil is also cooled, its resistive losses are quite negligible, more especially as the coil can then be superconducting. The signal to noise ratio is optimised by adjusting the number $n$ of turns of this coil and the operating frequency. From figure 2 , the signal to noise density ratio can be written as [6] :

$(S / N)^{s c}=\left|Y_{1}\right| e_{c}\left(\overline{e_{n R p}^{2}}\left|Y_{1}\right|^{2}+\overline{e_{n s c}^{2}}\left|\frac{1}{R_{1}}+j \frac{1}{L_{p} \omega_{0 p}}\left[\frac{\omega_{r}}{\omega_{0 p}}-\frac{\omega_{0 p}}{\omega_{r}}\right]\right|^{2}+\overline{i_{n s c}^{2}}+2 e_{n s c} i_{n s c} C_{I s c} \frac{1}{L_{p} \omega_{0 p}}\left[\frac{\omega_{r}}{\omega_{0 p}}-\frac{\omega_{0 p}}{\omega_{r}}\right]\right)^{-1 / 2}$

where $Y_{1}=1 / R_{1}+1 / j X_{1}=R_{p} /\left(R_{p}^{2}+L_{p}^{2} \omega_{r}^{2}\right)+L_{p} \omega_{r} / j\left(R_{p}^{2}+L_{p}^{2} \omega_{r}^{2}\right)$ and $\omega_{0 p}$ is the angular velocity corresponding to the resonance of the tank circuit. $\mathrm{C}_{\mathrm{Isc}}$ is the imaginary part of the correlation term between $e_{n s c}$ and $i_{n s c}$ (the real part is negligible). A cylindrical coil of volume $V_{c}$ with $n$ turns of meanelectrical cross section $s$ has an inductance $L_{p}=\mu_{0} K^{2} n^{2} / V_{c}=K_{2} n^{2}$, where $K$ is a numerical factor $(\sim 1)$ depending on the coil geometry [7]. The NMR signal is $e_{c}=\left(\mu_{0} \eta_{V} \Delta M s \omega_{r} n\right) / \sqrt{2}=K_{1} \omega_{r}^{2} n$, where $\eta_{V}$ is the filling factor of the coil and $\Delta \mathrm{M}$ the transverse spin magnetisation rotating at the angular velocity $\omega_{\mathrm{r}}$. The losses of the pick-up circuit (Fig. 1 and 2) act as an effective resistance $R_{p}[8]$ :

$R_{p}=n_{\text {sple }} \pi \mu_{0}^{2} \omega_{r}^{2} n^{2} b^{4} h_{\text {sple }} /\left[32 p\left(a^{2}+(h / 2)^{2}\right)\right]+R_{c}=K_{4} \omega_{r}^{2} n^{2}+R_{c}=R_{\text {sple }}+R_{c}$

where $h$ and a are respectively the height and the radius of the pick-up coil. The first term in (3) represents the inductive losses in the sample wires induced by the radiofrequency field, assuming that the skin effect and the dielectric losses are negligible. The connection and bonding resistances to the preamplifier involve an overall resistance, noted $R_{c}$, which is independent of the frequency. $R_{c}$ and $R_{p}$ bring thermal noise sources given by $\mathbf{e}_{n R c}$ and $e_{n R p}$. Analysis of expression (2) shows that to maximise the signal to noise 
density ratio, it is necessary to operate close to the resonant frequency of the tank circuit and to use an input circuit with a high quality factor. So, a high pick-up inductance and a low value for $R_{p}$ are needed. The value of the inductance $L_{p}$ is limited by the parasitic self capacitance of the coil so it is difficult to optimise the input impedance in the low frequency range.

In the particular case of the study of small size samples $\left(\leq 0.1 \mathrm{~cm}^{3}\right)$, like thin films, a reasonable filling factor with a large pick-up coil is not obtainable. The ratio S/N cannot be optimised; the NMR signal is weak and the noise generator $e_{n s i}$ dominates the other noise sources. It might therefore be interesting to consider the performances of a DC SQUID preamplifier located very close to the sample in the cryogenic environment.

\subsubsection{SQUID amplifier : selective configuration}

The optimal input impedance of a SQUID amplifier is low. Therefore, as shown in figure 1 , the use of a series resonant input circuit is possible. The signal to noise density ratio is given by [6]

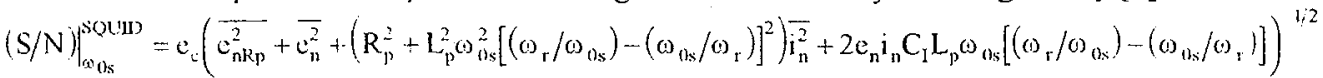

where $\omega_{0 s}$ is the resonant frequency of the input circuit $\left(R_{p}, L_{p}+L_{i}, C_{s}\right)$. Taking the derivative of $\left(\mathrm{S} /\left.\mathrm{N}\right|_{\omega_{0 s}} ^{\mathrm{SQUID}}\right)^{2}$ with respect to $\mathrm{n}$, the optimal pick-up inductance is expressed as

$\mathrm{L}_{\text {popts }}^{\text {SQUid }}=\mathrm{K}_{2} \mathrm{n}_{\mathrm{opts}}^{2}=\left(\mathrm{K}_{2} / \mathrm{i}_{\mathrm{n}}\right)\left[\left(\overline{\mathrm{e}_{\mathrm{nRc}}^{2}}+\overline{\mathrm{e}_{\mathrm{n}}^{2}}+\mathrm{R}_{\mathrm{c}}^{2} \overline{\mathrm{i}_{\mathrm{n}}^{2}}\right) /\left(\mathrm{K}_{4}^{2} \omega_{\mathrm{r}}^{4}+\mathrm{K}_{2}^{2} \omega_{0 \mathrm{~s}}^{2}\left[\left(\omega_{\mathrm{r}} / \omega_{0 \mathrm{~s}}\right)-\left(\omega_{0 \mathrm{~s} /} / \omega_{\mathrm{r}}\right)\right]^{2}\right)\right]^{1 / 2}$

As in section 1.2.1, operation close to the resonant frequency with a high quality factor is necessary. So, the NMR detection on small size samples is still difficult. In comparison with the use of semiconductor amplifiers, this configuration essentially allows to profit from the low energy $\left(e_{n} i_{n}\right)$ of the SQUID

\subsubsection{SQUID amplifier : broadband configuration}

The two configurations considered up to now are selective in frequency, that is an important limitation for some NMR experiments. Another arrangement is obtained by connecting the pick-up coil directly to the SQUID input coil $L_{i}\left(C_{s}\right.$ is suppressed in Fig. 1). In this case, the signal to noise density ratio (5) is written as :

$\mathrm{S} /\left.\mathrm{N}\right|^{\text {SQUiD }}=\mathrm{e}_{\mathrm{c}}\left(\overline{\mathrm{e}_{\mathrm{nRp}}^{2}}+\overline{\mathrm{e}_{\mathrm{n}}^{2}}+\left(\mathrm{R}_{\mathrm{p}}^{2}+\mathrm{L}_{\mathrm{p}}^{2} \omega_{\mathrm{r}}^{2}\right) \overline{\mathrm{i}}_{\mathrm{n}}^{2}+2 \mathrm{e}_{\mathrm{n}} \mathrm{i}_{\mathrm{n}} \mathrm{C}_{\mathrm{l}} \mathrm{L}_{\mathrm{p}} \omega_{\mathrm{r}}\right)^{-1 / 2}$

In this configuration, the optimal pick-up inductance is expressed as

$\mathrm{L}_{\text {popt }}^{\text {SQUid }}=\mathrm{K}_{2} \mathrm{n}_{\text {opt }}^{2}=\mathrm{K}_{2}\left[\left(\overline{\mathrm{e}_{\mathrm{nRc}}^{2}}+\overline{\mathrm{e}_{\mathrm{n}}^{2}}+\mathrm{R}_{\mathrm{c}}^{2} \overline{\mathrm{i}_{\mathrm{n}}^{2}}\right) /\left(\mathrm{K}_{4}^{2} \omega_{\mathrm{r}}^{2}+\mathrm{K}_{2}^{2}\right)\right]^{1 / 2} /\left(\omega_{\mathrm{r}} \mathrm{i}_{\mathrm{n}}\right)$

The maximum signal to noise density ratio becomes :

$S /\left.N\right|_{n=n_{\text {opt }}} ^{S Q U D}=K_{1} \omega_{r}^{2}\left(2 \omega_{r} i_{n}\left[\left(\overline{c_{n R c}^{2}}+e_{n}^{2}+R_{c}^{2} \bar{i}_{n}^{2}\right)\left(K_{4}^{2} \omega_{r}^{2}+K_{2}^{2}\right)\right]^{1 / 2}+4 k_{B} T K_{4} \omega_{r}^{2}+2 R_{c} K_{4} \omega_{r}^{2} \bar{i}_{n}^{2}+2 e_{n} i_{n} C_{1} K_{2} \omega_{r}\right)^{1 / 2}$

In the ideal case $\left(R_{p}=0\right), L_{\text {popt }}^{\text {SQUD }}(7)$ can be written as $L_{\text {popt }}^{\text {SQUI }}=L_{i}\left(11 \alpha_{c}^{4} / 16-3 \alpha_{c}^{2} / 2+1\right)$. A numerical evaluation based on the SQUID SQD 1002 from Conductus shows that this optimal inductance is quite low $\left(\mathrm{n}_{\mathrm{op}} \approx 3, \mathrm{~L}_{\mathrm{popt}}^{\mathrm{SQLID}} \approx 0,56 \mathrm{~L}_{\mathrm{i}} \approx 90 \mathrm{nH}\right)$. A reasonable value of the filling factor can even be obtained on small size samples.

\subsection{Comparison of coupling configurations}

Table 1 presents the signal-to-noise density ratio for the three configurations considered. The first two ones correspond to selective configurations, the last one to the broadband configuration

In the case of a selective input circuit, the dc SQUID based system is the most sensitive circuit for a given quality factor. It must be mentioned that in a pulsed NMR experiment, it is not possible to analyse for 
example copper samples with circuits which have a high quality factor $(Q \geq 1400)$ because of the bandwidth necessary to transmit the signal variation, since the spin-spin relaxation time $T_{2}$ of ${ }^{6.3} \mathrm{Cu}$ isotope is $77 \mu \mathrm{s}$. These circuits can however be used for other samples [1] or continuous NMR experiment. In most cases, the dc SQUID amplifiers offer better performances than semiconductor amplifiers. This is particularly evident for the small size samples where a broadband DC SQUID achieves an increase of more than $10 \mathrm{~dB}$ on the signal to noise density ratio compared to the selective semiconductor based configuration.

\begin{tabular}{|c|c|c|c|c|c|c|}
\hline \multirow[b]{2}{*}{$\begin{array}{l}\text { resistive losses } \\
(\Omega)\end{array}$} & \multicolumn{3}{|l|}{$\mathrm{h}_{\mathrm{splk}}=10 \mu \mathrm{m}$} & \multicolumn{3}{|c|}{$\mathrm{h}_{\text {solk }}=\mathrm{h} \cong \mathrm{cm}$} \\
\hline & $\begin{array}{l}R_{\text {sple }}=0 \\
R_{p}=R_{c}=0.1\end{array}$ & $\begin{array}{l}R_{\text {spic }}=0 \\
R_{p}=R_{c}=1\end{array}$ & $\begin{array}{l}R_{c}=0 \\
R_{r}=R_{\text {sple }}\left({ }^{*}\right)\end{array}$ & $\begin{array}{l}\mathrm{R}_{\text {sple }}=0 \\
\mathrm{R}_{\mathrm{p}}=\mathrm{R}_{\mathrm{c}}=0.1\end{array}$ & $\begin{array}{l}\mathrm{R}_{\text {sple }}=0 \\
\mathrm{R}_{\mathrm{p}}=\mathrm{R}_{\mathrm{c}}=1\end{array}$ & $\begin{array}{l}\mathrm{R}_{\mathrm{c}}=0 \\
\mathrm{R}_{\mathrm{p}}=\mathrm{R}_{\text {sple }}\left({ }^{\circ}\right)\end{array}$ \\
\hline $\begin{array}{l}L_{p} \\
\text { quality factor } \\
S /\left.N\right|^{s i}(d B)\end{array}$ & $\begin{array}{l}L_{\text {pmax }} \\
14000 \\
59\end{array}$ & $\begin{array}{l}L_{\text {pmax }} \\
1400 \\
56\end{array}$ & $\begin{array}{l}L_{\text {pmax }} \\
13800 \\
57\end{array}$ & $\begin{array}{l}L_{\text {pmax }} \\
14000 \\
119\end{array}$ & $\begin{array}{l}L_{\text {pmax }} \\
1400 \\
116\end{array}$ & $\begin{array}{l}L_{\text {pmax }} \\
13.8 \\
105\end{array}$ \\
\hline $\begin{array}{l}L_{\text {popt }}^{S Q U I D} \\
\text { quality factor } \\
S /\left.N\right|_{\omega_{0 .}} ^{S Q U I D} \text { (dB) }\end{array}$ & $\begin{array}{l}L_{\text {pinax }} \\
14000 \\
78\end{array}$ & $\begin{array}{l}\mathrm{L}_{\mathrm{pmax}} \\
1400 \\
68\end{array}$ & $\begin{array}{l}L_{\text {pilax }} \\
13800 \\
78\end{array}$ & $\begin{array}{l}L_{\text {max }} \\
14000 \\
138\end{array}$ & $\begin{array}{l}\mathrm{I}_{\operatorname{mixx}} \\
1400 \\
128\end{array}$ & $\begin{array}{l}L_{\text {pmax }} \\
13.8 \\
107\end{array}$ \\
\hline $\begin{array}{l}\mathrm{L}_{\text {pupt }}^{\text {SQUID }}(\mu \mathrm{H}) \\
S /\left.N\right|_{n=n_{\text {cat }}} ^{\text {SQCTD }}(d B)\end{array}$ & $\begin{array}{l}5.16 \\
72\end{array}$ & $\begin{array}{l}16.3 \\
67\end{array}$ & $\begin{array}{l}0.09 \\
93.4\end{array}$ & $\begin{array}{l}5.16 \\
112\end{array}$ & $\begin{array}{l}5.16 \\
107\end{array}$ & $\begin{array}{l}0.09 \\
115\end{array}$ \\
\hline
\end{tabular}

Table 1 : Comparison of theoretical signal to noise density ratio for different configurations of a pulsed NMR experiment at ${ }_{\mathrm{r}} / 2 \pi=\omega_{\mathrm{op}} / 2 \pi=\omega_{0 \mathrm{~s}} / 2 \pi=250 \mathrm{kHz}$ and $\mathrm{T}=4,2 \mathrm{~K}$. The copper sample $\left(\mathrm{n}_{\text {sple }}=125,000 ; \mathrm{b}=10 \mu \mathrm{m}\right)$ has a specific resistivity $\rho \approx 8.510^{-11} \Omega \mathrm{m}$. The radius of the pick-up coil is $a=0.5 \mathrm{~cm}$ and the chosen maximal value of $\mathrm{L}_{\mathrm{p}}$ is $L_{\text {pmax }}=0.89 \mathrm{mH}(n=300)$ which is imposed by the operating frequency. $R_{\text {sple }}{ }^{(*)}$ is the resistive losses of the part of the sample included in the pick-up coil. The selected semiconductor amplifier (JFET) has been optimised for a NMR experience at $250 \mathrm{kHz}$. [7] : $\mathrm{e}_{\mathrm{nsc}}=1.56 \mathrm{nV} / \sqrt{\mathrm{Hz}}, \mathrm{i}_{\mathrm{nsc}}=38 \mathrm{fA} / \sqrt{\mathrm{Hz}}$.

\section{HETERODYNE AMPLIFICATION WITH A DC SQUID}

In section 1, we have shown that DC SQUID based amplifiers offer better performances, even for the broadband coupling, than the semiconductor amplifiers. A key point for the realisation of a DC SQUID NMR receiver lies in the noise matching of the output of the SQUID amplifier to the subsequent semiconductor based stage. A matching device is then required. Two solutions are widely known. In the first one, which is broadband type, an up conversion transformer is inserted between the SQUID output and the FET input [9]. In the second one, a LC circuit is placed as shown in figure 3 ; as a consequence, the receiver becomes selective even if the pick-up coil coupling circuit is non selective. We now present in details the performances of a DC SQUID preamplifier-mixer which combines good noise properties and easy matching to the subsequent stages and is well adapted to continuous NMR experiments. Since, it may operate as a wide band at NMR frequencies. Let us examine, on figure 3, how the system works.

\subsection{Principle of the Method}

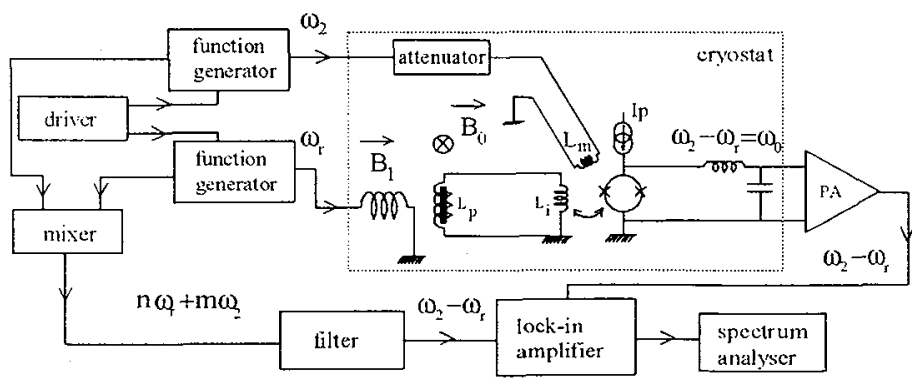

Figure 3 : Schematics of the spectrometer with a de SQUID for amplifying the NMR signal. 
In continuous NMR experiments, two perpendicular magnetic field $\overrightarrow{\mathrm{B}}_{0}$ (static) and $\overrightarrow{\mathrm{B}}_{1}$ (pulsation $\omega_{\mathrm{r}}$ ) are simultaneously applied to the sample. A NMR signal $e_{c}$ is then induced at $\omega_{r}$, amplitude and phase depending on the angular velocity difference between $\omega_{r}$ and the Larmor one. Thus, in continuous NMR experiments, the $\omega_{\mathrm{r}}$ value in slowly swept over the frequency band of interest in order to detect the different resonance peaks. In the system shown in figure 3 , a second high level sinusoidal magnetic field is directly applied at pulsation $\omega_{2}$ to the SQUID. So, due to its non-linear characteristic, the SQUID output signal contains components at pulsation $n \omega_{r}+m \omega_{2}\left(n, m\right.$ are integer) where only one term $\left(\omega_{2}-\omega_{r}\right.$ in this application) develops voltage at the FET input. The two function generators, driven by a computer, have therefore to be swept synchronously in order to maintain the $\omega_{2}-\omega_{\mathrm{r}}$ constant, equal to the resonant pulsation of the LC matching circuit. The signal $\left(a t \omega_{2}-\omega_{\mathrm{f}}\right)$ is amplified at room temperature and lock-in detected to restore the SQUID signal. The lock-in amplifier is triggered by the function generators outputs via a mixer and a filter. The modulating signal of pulsation $\omega_{2}$ and amplitude $\alpha \Phi_{0}(\alpha \approx 0.25)$ is centred on $\Phi_{0} / 2$ and injected by the modulation coil $L_{m}$. We propose a theoretical model based on a simplified sinusoidal voltage to flux characteristic of the DC SQUID to evaluate the performances of our system [4]

$V_{s}(t)=v_{n}(t)+e_{n a}(t)+A\left(1-\cos \left[\frac{2 \pi}{\Phi_{0}}\left(\Phi_{B}+\alpha \Phi_{0} \cos \left(\omega_{2} t+\theta_{n}(t)\right)+\Delta \Phi \cos \left(\omega_{1} t+\delta\right)+\varphi_{n}(t)\right)\right]\right)$

where $\mathrm{A}$ is the signal amplitude at the DC SQUID output, $\Phi_{\mathrm{B}}$ is the bias flux, $\Delta \Phi$ the amplitude of the signal which is assumed to be sinusoidal. The main sources of noise in our experimental set-up are the SQUID intrinsic noise and the equivalent noise voltage $e_{n a}(t)$ of the semiconductor amplifier referred to the SQUID output. As usual, the SQUID intrinsic noise is supposed to be only due to the Johnson noise in the resistive shunts of the junctions. The SQUID is affected in two ways : a voltage noise $v_{n}(t)$ is induced in series with the SQUID and a flux noise $\varphi_{n}(t)$ is applied to the SQUID inductance $L_{s}$. Furthermore, a phase noise $\theta_{n}(t)$ can also be introduced by the synthesised generator which supplies the pump signal.

The ratio of the flux-voltage transfer to the effective voltage noise across the SQUID at the pulsation $\omega_{0}$ is given by [4] :

$\frac{\partial \overline{\mathrm{V}}_{\mathrm{s}} / \partial \Phi_{\mathrm{a}}}{\sqrt{\frac{\mathrm{V}_{\mathrm{sb}}^{2}\left(\omega_{0}\right)}{1}}}=2 \pi \mathrm{AJ}_{1}(2 \pi \alpha)\left(\overline{\mathrm{v}_{\mathrm{n}}^{2}\left(\omega_{0}\right)}+\overline{\mathrm{e}_{\mathrm{na}}^{2}\left(\omega_{0}\right)}+2\left\{\pi \mathrm{AJ}_{1}(2 \pi \alpha)\right\}^{2}\left\{\left(\frac{2}{\Phi_{0}}\right)^{2} \overline{\varphi_{\mathrm{n}}^{2}\left(\omega_{2}-\omega_{0}\right)}+\alpha^{2} \overline{\theta_{\mathrm{n}}^{2}\left(2 \omega_{2}-\omega_{0}\right)}\right\}\right)^{-1 / 2}$

The flux-voltage transfer coefficient is maximal for $\alpha \approx 0,29$ and $\Phi_{\mathrm{B}}=\mathrm{k} \Phi_{0} / 2$ with $\mathrm{k}$ integer. From the expression relative to a direct measurement, the flux-voltage transfer coefficient is equal to $2 \pi A$ when $\Phi_{\mathrm{B}}=(\mathrm{l} / 4+\mathrm{k} / 2) \Phi_{0}$. The application of flux modulation decreases this value of a factor $J_{1}(2 \pi \alpha)=0.58$ for $\alpha=0.29$. But, when an appropriate resonant circuit is associated with a low noise amplifier, $\overline{\mathrm{e}_{\mathrm{na}}^{2}\left(\omega_{0}\right)}$ is very small compared to the other noise sources (10). In the opposite, term $\overline{\mathrm{e}_{\mathrm{na}}^{2}\left(\omega_{1}\right)}$, obtained in direct mode, is not negligible when the application requires a wide band preamplifier. Furthermore, we have verified that a low noise synthesised generator can make $\overline{\theta_{n}^{2}(\omega)}$ negligible.

\subsection{Experimental device and measurements}

The SQUID used is a commercial DC SQUID (Conductus SQD1075), a Jaycox and Ketchen's type. The relevant characteristics measured at $T=4.2 \mathrm{~K}$ are $: R=5 \Omega, L_{s}=170 \mathrm{pH}, L_{i}=100 \mathrm{nH}$ and $I_{c}=12.5 \mu \mathrm{A}$. The performances of the SQUID amplifier have been studied when a sinusoidal pump signal at frequencies ranging from few hundred $\mathrm{kHz}$ up to a few $\mathrm{MHz}$ is applied. For example in Fig. 4.b), the signal to be analysed has been simulated by a small sinusoidal signal of amplitude $\Phi_{0} / 400$ at $\omega_{\mathrm{r}} / 2 \pi=500 \mathrm{kHz}$.

The best flux sensitivity of the SQUID measured in the direct mode, $1.5 \mu \Phi_{0} / \sqrt{\mathrm{Hz}}$ at $\mathrm{i}_{\mathrm{p}}=1.5$ (Fig. 4.a).), corresponds to an energy resolution $\varepsilon=70 \mathrm{~h}$. In the heterodyne mode, the best flux sensitivity $5.3 \mu \Phi_{0} / \sqrt{\mathrm{Hz}}$ i.e. $\varepsilon=880 \mathrm{~h}$, is deteriorated by a factor close to 3 that corresponds to the ratio of the flux voltage transfer coefficients. According to our theoretical model, the flux - voltage transfer coefficient $V_{(1)}$ 
should only be decreased by a factor $\mathrm{J}_{1}(2 \pi \alpha) \approx 0.58$ in the pumped mode. Experimentally, some irregularities of the $\mathrm{V}(\Phi)$ characteristic are used to increase the output signal in the direct mode whereas the pumped mode (sweeping around $0.5 \Phi_{0}$ ) implies that the transfer coefficient results from an averaging of the slopes of the $V(\Phi)$ curve. For the same reason, the coefficient $V_{\Phi}$ observed in the pumped mode is close to the expected theoretical value.
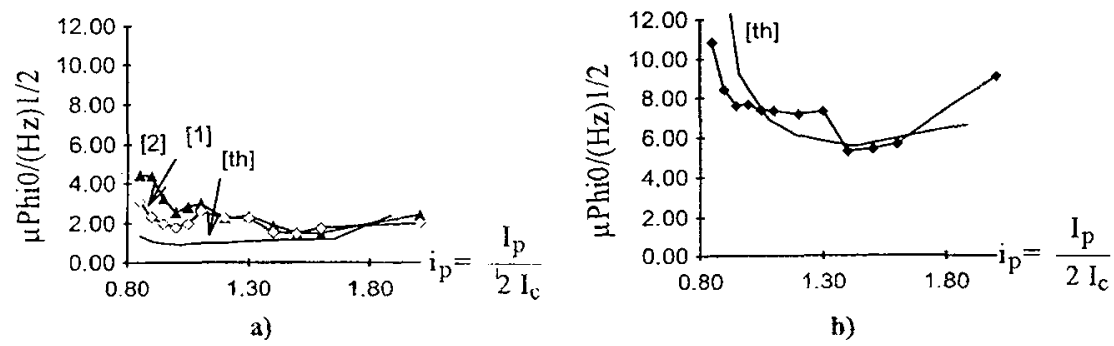

Figure + : SQUID flux sensitivity : a) Direct measurement at $35 \mathrm{kHz}$ for an applied flux of $0.25 \Phi_{0}[1]$ and, $0.75 \Phi_{0}[2]$ plotted with semi-theoritical [th] and experimental curves. These semi-theoretical performances are calculated from voltages noise and amplitudes $A$ of the experimentally measured output signal. b) Measurement with pump signal at $535 \mathrm{kHz}$ for an applied flux of $0.5 \Phi_{0}$ plotted with semi-theoritical.[th] and experimental curves ; $\omega_{\mathrm{r}} / 2 \pi=500 \mathrm{kHz}$

\section{CONCLUSION}

We have compared the performances of DC SQUID and semiconductor amplifiers for the spectral analysis of a NMR signal in the low frequency range. Our calculations clearly show that SQUID amplifiers are the most sensitive amplifiers for the NMR detection of cooled samples but they are difficult to use. So, DC SQUID preamplifier will be only used for small size samples. The most interesting configuration is obtained when the SQUID amplifier is associated with a broadband input stage.

We have determined the optimal input impedance of SQUID amplifier and we propose an amplification method which may operate over a large frequency range and matches the impedance of the SQUID output. This method takes advantage of the flux modulation techniques and translates the signal spectrum so as to operate at a low fixed intermediate frequency signal.

\section{References}

[1] Fan N.Q., Heaney M.B., Clarke J., Newitt D., et al, IEEE Trans. Mag. 25, 2 (1989) 1193-1 199

[2] Freeman M.R., Germain R.S., Richarson R.C., et al, Appl. Phys. Lett. 48, 4 (1986) 300-302

[3] Narasimhan L.R., Takigawa M., Ketchen M.B., Appl. Phys. Lett. 65, n¹0 (1994) $1305-1307$

[4] Hubert O., Monfort Y., Lepaisant J., Gunther C., Bloyet D., J. Phys. III France 6 (1996) 77-90

[5] Bloyet D., Gunther C., Techriques de l'ingénieur (in french), to be published in May 1996

[6] Hubert O., Ph. D. (in french), University of Caen (1995)

[7] Lepaisant J., Bloyet D., Varoquaux E., Rev. Sci. Instrum., 55, n 4 (1984) 521-526

[8] Gadian D.G., Robinson F.N.H., J. Mag. Reson., 34 (1979) 449-455

[9] Drung D., Sci. Technol 4 (1991) 377-385 